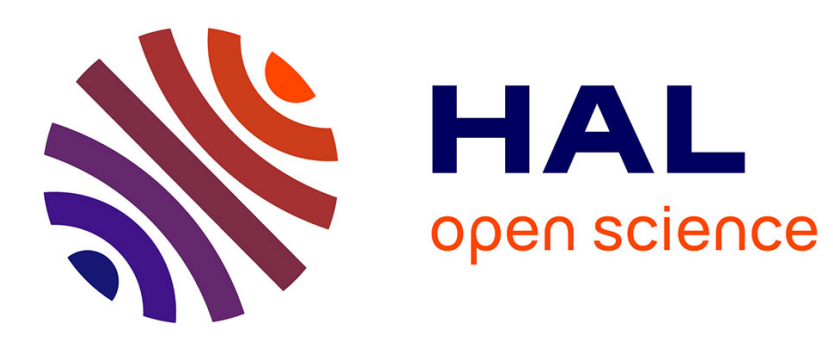

\title{
Création et immobilisation de fonctions chloramine et/ou bromamine sur une anode de dioxyde d'étain en eau de mer
}

\author{
Catherine Debiemme, Sanae Haskouri, J. L Duval, Hubert Cachet
}

\section{To cite this version:}

Catherine Debiemme, Sanae Haskouri, J. L Duval, Hubert Cachet. Création et immobilisation de fonctions chloramine et/ou bromamine sur une anode de dioxyde d'étain en eau de mer. Matériaux \& Techniques, 2006, 94, pp.433 - 439. 10.1051/mattech:2007018 . hal-01729195

\section{HAL Id: hal-01729195 \\ https://hal.sorbonne-universite.fr/hal-01729195}

Submitted on 12 Mar 2018

HAL is a multi-disciplinary open access archive for the deposit and dissemination of scientific research documents, whether they are published or not. The documents may come from teaching and research institutions in France or abroad, or from public or private research centers.
L'archive ouverte pluridisciplinaire HAL, est destinée au dépôt et à la diffusion de documents scientifiques de niveau recherche, publiés ou non, émanant des établissements d'enseignement et de recherche français ou étrangers, des laboratoires publics ou privés. 


\title{
Création et immobilisation de fonctions chloramine et/ou bromamine sur une anode de dioxyde d'étain en eau de mer
}

\author{
C. Debiemme-Chouvy' ${ }^{1}$, S. Haskouri' ${ }^{1}$, J.L. Duval ${ }^{2}$ et H. Cachet ${ }^{1}$ \\ 1 CNRS-UPR 15, Laboratoire Interfaces et Systèmes Électrochimiques (LISE), Université Pierre et Marie \\ Curie - Paris, France \\ 2 UMR CNRS 6600 - Université de Technologie de Compiègne, Laboratoire de Biomécanique et Génie \\ Médical, Compiègne, France \\ e-mail : huc@ccr.jussieu.fr
}

\section{Mots-clés :}

Dioxyde d'étain; protéines; film antibactérien; chloramine

\begin{abstract}
Résumé - Les agents oxydants sont utilisés couramment pour prévenir la formation de biosalissures. Ils peuvent être injectés au voisinage de la surface à protéger ou mieux, immobilisés sur cette surface. Dans ce travail, nous montrons le caractère antibactérien acquis par le dépôt organique formé par modification chimique d'une protéine, l'albumine du sérum bovin (BSA), par de l'acide hypochloreux et/ou hypobromeux, électrogénérés à la surface d'une anode de dioxyde d'étain. Les milieux électrolytiques considérés sont des solutions de chlorure de sodium et d'eau de mer naturelle. Les études électrogravimétriques, les observations par microscopie électronique à balayage, complétées par des analyses de surface, montrent la présence d'un film organique épais contenant les éléments chlore et/ou brome, suivant le milieu. Dans ce film, la BSA conserve sa structure primaire, mais subit des modifications chimiques. La stabilité du film organique est améliorée par la présence de cations bivalents comme $\mathrm{Ca}^{2+}$ et $\mathrm{Mg}^{2+}$. Il a été montré antérieurement que les éléments $\mathrm{Cl}$ et $\mathrm{Br}$ étaient incorporés sous forme de fonctions chloramine et/ou bromamine. L'efficacité antibactérienne a été établie en observant la non colonisation de la surface de $\mathrm{SnO}_{2}$ traitée, par une souche de bactéries Escherichia coli, par microscopie à fluorescence.
\end{abstract}

\section{Key words:}

Tin dioxide; proteins; antibacterial film; chloramine
Texte reçu le 12 juin 2007 accepté le 20 juin 2007

\begin{abstract}
Creation and immobilisation of chloramine and/or bromamine groups on tin dioxide anodes in seawater. Oxidising agents are currently used to prevent biofouling in two ways, either by injection near the surface to be protected, or better, to be immobilized on the very surface. In this work, it is shown that the organic deposit formed by chemical oxidation of a protein, the bovin serum albumin (BSA), by hypochlorous and/or hypobromous acids, electrogenerated at a tin dioxide anode, presents antibacterial properties. Sodium chloride or natural seawater have been considered as reaction media. Electrogravimetric measurements, scanning electron microscopy observations completed by surface analyses, point out that a chlorine and/or bromine containing, thick organic deposit is formed at the $\mathrm{SnO}_{2}$ surface. In the film, BSA molecules keep their primary structure but undergo chemical modifications. Film stability was found to be improved in the presence of bivalent cations as $\mathrm{Ca}^{2+}$ and $\mathrm{Mg}^{2+}$. It was shown previously that $\mathrm{Cl}$ and $\mathrm{Br}$ elements are incorporated as chloramine and bromamine groups, respectively. The antibacterial efficiency was demonstrated by observing the surface colonisation by Escherichia coli bacteria with fluorescence microscopy, in the presence or not of the chlorinated (brominated) BSA deposit.
\end{abstract}

our limiter le développement de biosalissures, on a recours à des produits chimiques, toxiques vis-à-vis des bactéries et autres microorganismes. Parmi ces produits biocides, se trouvent les agents oxydants qui agissent chimiquement sur la paroi cellulaire des micro-organismes en perturbant leur métabolisme. Comme biocides oxydants [1], on peut citer le chlore $\left(\mathrm{Cl}_{2}\right)$ et ses dérivés (acide hypochloreux, hypochlorite de sodium, hypochlorite de calcium, monochloramine, dichloroisocyanurate de sodium), le brome et ses dérivés (acide hypobromeux, hypobromite et bromamine), dioxyde de chlore $\left(\mathrm{ClO}_{2}\right)$ et l'ozone $\left(\mathrm{O}_{3}\right)$.

La formule générale des chloramines est $>\mathrm{N}-\mathrm{Cl}$ : l'atome de chlore possède un caractère oxydant puisqu'il est au degré d'oxydation (+I) [2]. 
Les chloramines diffusent lentement le chlore, entraînant une désinfection moins agressive que celle produite par l'acide hypochloreux, $\mathrm{HOCl}$. Lorsque le chlore est utilisé comme désinfectant, l'odeur et la saveur de l'eau ont ainsi été améliorées grâce à l'utilisation de chloramines. Les chloramines sont aussi utilisées pour la désinfection des eaux usées, pour celle de l'eau de consommation, et pour la prévention des biofilms dans les systèmes de refroidissement. Les travaux de Momba et al. [3, 4] montrent le rôle important des chloramines pour le traitement des eaux et son efficacité pour empêcher la croissance et l'accrochage des bactéries.

Les chloramines peuvent être formées lors d'une réaction entre de la matière organique se trouvant dans le milieu et l'acide hypochloreux. De très nombreuses études, dans le domaine biomédical, se sont intéressées à l'interaction entre $\mathrm{HOCl}$ et les protéines. Cette interaction serait, en particulier, à l'origine des premiers stades de développement de l'arthériosclérose. En effet, la myéloperoxidase (enzyme produite par les polynucléaires neutrophiles) catalyse la réaction entre $\mathrm{H}_{2} \mathrm{O}_{2}$ et les ions chlorure, conduisant à la formation de $\mathrm{HClO}$ [5-7], lequel, en excès, peut provoquer des lésions des tissus [8]. Winterbourn et al. [9], puis Hawkins et al. [10-12] ont montré que l'oxydation de protéines par $\mathrm{HClO}$ entraîne notamment la formation de groupements chloramine. Ces auteurs ont également montré que l'acide hypochloreux interagit préférentiellement avec les chaînes latérales contenant des fonctions amine primaire, par exemple la lysine, créant des liaisons $\mathrm{N}-\mathrm{Cl}$ conduisant donc à la formation de groupements chloramine portés par le squelette de la protéine, eux mêmes pouvant évoluer vers la formation de radicaux centrés sur un atome d'azote. Il est à noter que le brome joue un rôle analogue à celui du chlore, l'interaction avec la matière organique conduisant à la formation de groupements bromamine ayant des propriétés oxydantes analogues à celles des chloramines.

Dans ce travail, nous nous intéressons à la formation et à l'action antibactérienne de groupements chloramine et bromamine, en vue d'empêcher la formation d'un biofilm sur une fenêtre de verre immergée en milieu marin. Nous avons montré antérieurement que la formation de tels groupements oxydants pouvait être obtenu à la surface d'un film transparent de dioxyde d'étain ayant été polarisé à $+1,5 \mathrm{~V} / \mathrm{ECS}$, au contact d'un milieu contenant des ions chlorure et des protéines comme l'albumine du sérum bovin (BSA) [13]. Dans ces conditions de polarisation, de l'acide hypochloreux est produit par oxydation électrochimique des ions chlorure. Il réagit chimiquement avec les protéines. Ces dernières s'agrégent à la surface de $\mathrm{SnO}_{2}$ et, en même temps, des fonctions amine portées par des chaînes latérales sont modifiées en fonctions chloramine [14]. Dans cet article, nous présentons plus particulièrement les résultats obtenus en eau de mer naturelle, l'aspect des surfaces modifiées après traitement électrochimique en présence de BSA et l'efficacité biocide de la chloration du film organique ainsi formé visà-vis d'une souche bactérienne d'Escherichia coli.

\section{Conditions expérimentales}

Les films de dioxyde d'étain sont synthétisés par pyrolyse d'un aérosol sur des lames de verre ou sur des quartz piézoélectriques pour les mesures d'électrogravimétrie (coupe AT - $6 \mathrm{MHz}$ - coefficient de sensibilité fréquence/masse : $-75 \mathrm{~Hz} \mu \mathrm{g}^{-1} \mathrm{~cm}^{2}$ [15]). Dans le contexte des applications antisalissures, les films $\mathrm{SnO}_{2}$ sont constitués d'une sous-couche dopée au fluor, recouverte d'une couche dopée à l'antimoine, chaque couche ayant une épaisseur de $300 \mathrm{~nm}$ [16]. La résistance carré est de l'ordre de 10 ohms. Selon les expériences, nous avons solubilisé de la BSA (Sigma) (masse moléculaire : $66 \mathrm{kD}$, taille : $14 \times 4 \times 4 \mathrm{~nm}^{3}$ ) dans une solution aqueuse de $\mathrm{NaCl} 0,5 \mathrm{M}$, ou dans de l'eau de mer naturelle, à des concentrations variant de 0,05 à $0,5 \%$. Les mesures électrochimiques et électrogravimétriques ont été effectuées dans une cellule à trois électrodes de volume $20 \mathrm{~cm}^{3}$. Tous les potentiels sont mesurés par rapport à une électrode de référence au calomel en solution saturée de $\mathrm{KCl}$ (ECS).

Les surfaces traitées ont été imagées à l'aide d'un microscope électronique à balayage LEO Stereoscan 440 couplé à un dispositif EDX d'analyse en énergie des rayons $X$ réémis (PGT - Spirit).

L'étude de l'adhésion et de la croissance bactérienne a été effectuée à l'aide d'une souche d'Escherichia coli (E. coli) [17]. Les cultures ont été réalisées dans des boîtes de Pétri de diamètre $90 \mathrm{~mm}$, sur lamelles de verre recouvertes par un film de $\mathrm{SnO}_{2}$. La concentration bactérienne initiale est de 
l'ordre de 2 à $5 \times 10^{7}$ bactéries par $\mathrm{mL}$ dans $\mathrm{NaCl}$ 0,5 M. Le volume de suspension bactérienne est de $21 \mathrm{~mL} /$ boîte. Après 17 heures $\mathrm{d}$ 'incubation, les lames de verre ont été rincées trois fois à la verticale avec $\mathrm{NaCl} 0,5 \mathrm{M}$, puis égouttées en vue des observations par microscopie à fluorescence. La solution bactérienne est préparée dans un bouillon de culture en solubilisant dans de l'eau distillée $8 \mathrm{~g} / \mathrm{L}$ de peptone (Nutrient Broth de «DIFCO » Réf. 234000), puis mise à l'autoclave, ajout d'une suspension d'E. coli décongelée à raison de $5-8 \mathrm{~mL} / 500 \mathrm{~mL}$ de bouillon, puis incubation $17 \mathrm{~h}$ à $37^{\circ} \mathrm{C}$.

Le principe d'identification des bactéries mortes ou vivantes repose sur l'incorporation sélective de sondes fluorescentes qui marquent l'ADN des bactéries. Selon l'état des parois, la sonde pénètre ou pas. Deux sondes ont été utilisées. La première est l'iodure de propidium. Il est excitable à $488 \mathrm{~nm}$ et réémet de la fluorescence rouge. La seconde est l'acridine orange. Il est excitable à $490 \mathrm{~nm}$ et réémet de la fluorescence verte. Les observations s'effectuent après l'ajout de volumes égaux des solutions d'acridine orange et d'iodure de propidium. Le microscope à fluorescence utilisé est de type Leica DMLB, équipé d'un appareil photo numérique Nikon Coolpix 995.

\section{Viabilité des bactéries sur le film $\mathrm{SnO}_{2}$ après polarisation anodique en l'absence de BSA}

Sous polarisation anodique et en l'absence de BSA, le dégagement d'acide hypochloreux protège en continu la surface du film $\mathrm{SnO}_{2}$ contre les biosalissures. Il s'agit là d'un mode de protection actif lié à la production même de $\mathrm{HOCl}$. Toutefois, cette étape ne confère pas à la surface de $\mathrm{SnO}_{2}$ des propriétés antisalissures comme le montre la figure 1, quel que soit le milieu, artificiel ou naturel. Les images de coloration ont été obtenues par microscopie à fluorescence après 17 heures d'incubation dans un milieu nutritif en présence de $5 \times 10^{7}$ bactéries $/ \mathrm{mL}$, sur des surfaces $\mathrm{SnO}_{2}$ polarisées à $+1,5 \mathrm{~V} / \mathrm{ECS}$ pendant $1 \mathrm{~h} 30$ dans $\mathrm{NaCl}$ 0,5 M (Fig. 1a) et dans de l'eau de mer naturelle (Fig. 1b). Les deux surfaces de film $\mathrm{SnO}_{2}$ ayant subi une polarisation anodique ne montrent aucun caractère anti-bactérien, en les comparant avec une surface non traitée (Fig. 1c). La densité bactérienne est sensiblement la même quel que soit l'échantillon.

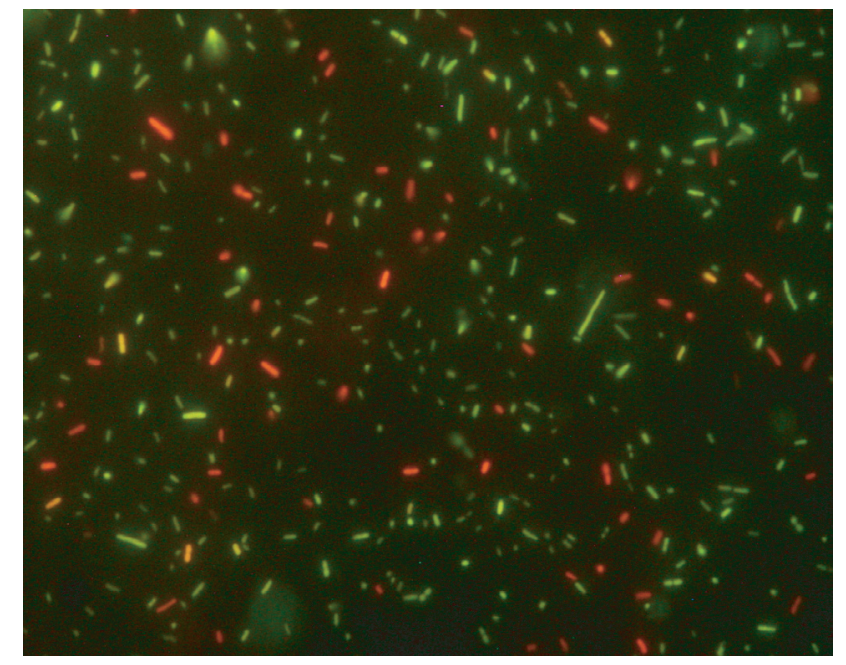

(a)

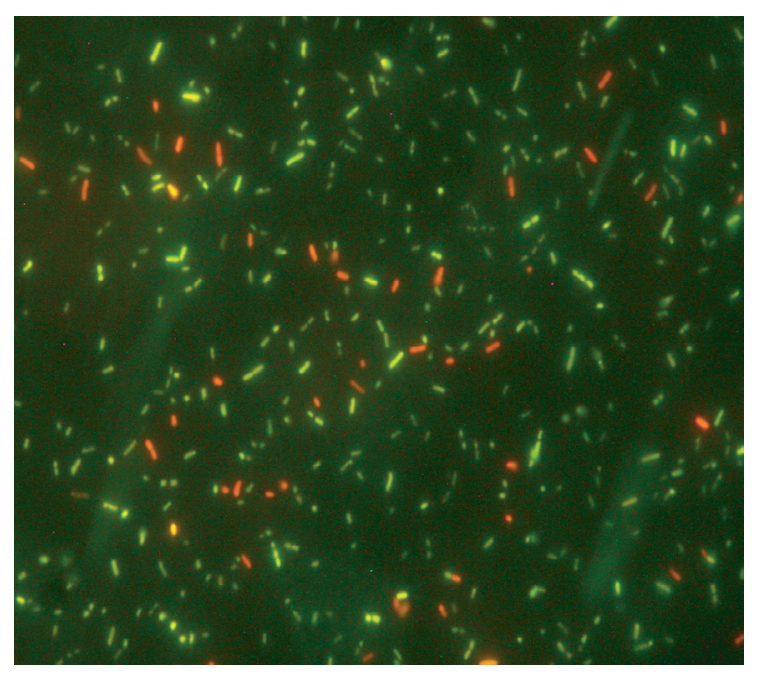

(b)

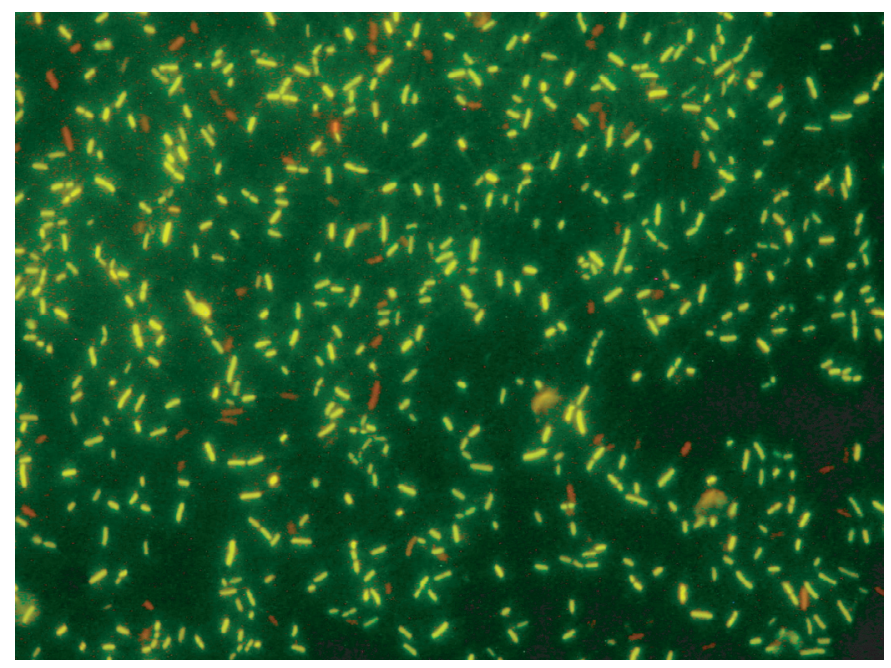

(c)

Fig. 1. Images de bactéries $E$. coli obtenues par microscopie à fluorescence : a) $\mathrm{NaCl} 0,5 \mathrm{M} 1 \mathrm{~h} 30, \mathrm{~b})$ eau de mer $1 \mathrm{~h} 30$ et c) témoin $\mathrm{SnO}_{2}$.

Fig. 1. Fluorescence microscopy images of E. coli bacteries: a) $\mathrm{NaCl}$ $0.5 \mathrm{M} 1 \mathrm{~h} 30 ; b)$ natural seawater $1 \mathrm{~h} 30$; c) $\mathrm{SnO}_{2}$ reference sample. 


\section{Réponses électrogravimétriques de films $\mathrm{SnO}_{2}$ en présence de BSA}

Comme le montre la figure 2, les molécules de BSA s'adsorbent spontanément sur la surface d'un film $\mathrm{SnO}_{2}$ au potentiel d'abandon. L'exemple présenté ici se réfère au cas de l'eau de mer naturelle. L'adsorption a lieu dès la première minute de mise en contact, la variation de masse du film $\mathrm{SnO}_{2}$ atteignant $0,8 \mu \mathrm{g} / \mathrm{cm}^{2}$. En faisant $\mathrm{l}^{\prime}$ hypothèse que la structure tertiaire de la BSA est conservée, la quantité théorique de BSA pour former une monocouche est de $1,8 \times 10^{12}$ molécules par $\mathrm{cm}^{2}$, soit $0,2 \mu \mathrm{g} \cdot \mathrm{cm}^{-2}$ pour une adsorption parallèle à la surface, et de $6,25 \times$ $10^{12}$ molécules par $\mathrm{cm}^{2}$, soit $0,7 \mu \mathrm{g} \cdot \mathrm{cm}^{-2}$, pour une adsorption perpendiculaire à la surface. Ainsi, en milieu eau de mer naturelle, les molécules de BSA seraient plutôt organisées perpendiculairement à la surface, la quantité adsorbée restant de l'ordre de la monocouche. Une telle organisation en milieu eau de mer naturelle pourrait provenir de la présence des cations bivalents $\left(\mathrm{Mg}^{2+}\right.$ et $\mathrm{Ca}^{2+}$ ) connus comme éléments stabilisants de la couche protéique adsorbée sur substrat en acier inoxydable [18]. Cet effet stabilisant est bien mis en évidence dans les réponses chronoélectrogravimétriques illustrées dans les figures $3 \mathrm{a}-\mathrm{c}$. La figure $3 \mathrm{a}$ montre la formation du film organique de BSA lorsque le potentiel de l'électrode est maintenu à $+1,5 \mathrm{~V} / \mathrm{ECS}$ en milieu $\mathrm{NaCl}$ 0,5 M+BSA à $0,3 \%$. Après coupure de la polarisation, on observe une perte de masse régulière indiquant un manque de stabilité de la couche protéique déposée. La situation est différente en présence des cations $\mathrm{Ca}^{2+}$ (Fig. 3b) ou $\mathrm{Mg}^{2+}$ (Fig. 3c). Les réponses chronoélectrogravimétriques ont été enregistrées dans des solutions $\mathrm{NaCl}+\mathrm{CaCl}_{2}$ et $\mathrm{NaCl}+\mathrm{MgCl}_{2}$, aux concentrations respectives existant dans l'eau de mer, en présence de BSA à $0,3 \%$. Il n'y a plus perte de masse après coupure du potentiel anodique. Le film organique apparaît stabilisé par la présence de cations bivalents comme ceux présents dans l'eau de mer. Ce résultat peut expliquer la stabilité remarquable obtenue pour le film de BSA formé en eau de mer naturelle [13], propriété cruciale pour des applications antifouling.

En complément de ces études, on a constaté que la quantité de matière protéique déposée sur une électrode de $\mathrm{SnO}_{2}$ polarisée à $+1,5 \mathrm{~V} / \mathrm{ECS}$ dépendait de la concentration volumique en BSA et de la valeur

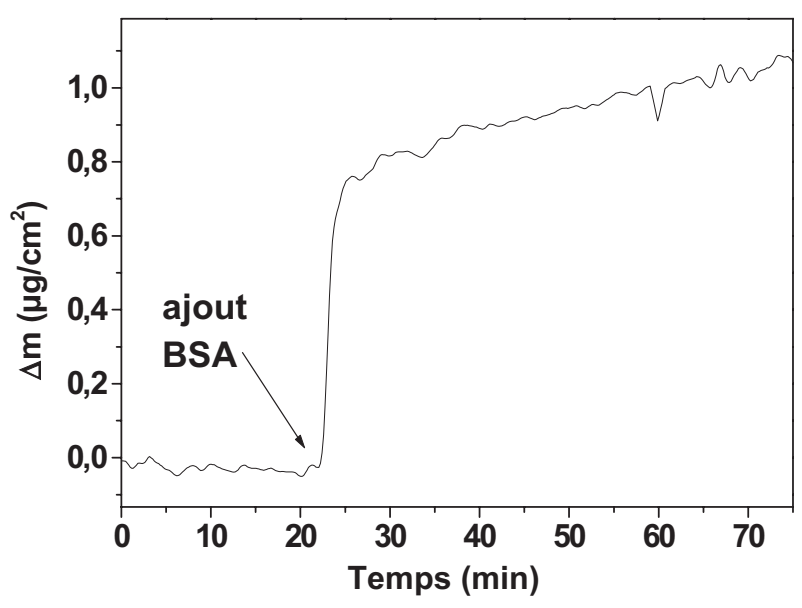

Fig. 2. Variation en fonction du temps de la masse d'une électrode $\mathrm{SnO}_{2}$ simplement immergée dans de l'eau de mer naturelle en présence de $0,5 \%$ de BSA.

Fig. 2. Time evolution of mass of a $\mathrm{SnO}_{2}$ electrode simply immersed in seawater in the presence of $0.5 \%$ BSA.

de la densité du courant anodique au point de fonctionnement. Des expériences menées jusqu'à présent, on peut conclure qu'il faut maintenir un rapport des concentrations [HClO]/[BSA] suffisamment élevé pour que les molécules de protéines puissent interagir efficacement avec celles de $\mathrm{HClO}$ produites électrochimiquement.

\section{Propriétés des films de BSA électrodéposés}

L'aspect des films organiques formés de BSA ayant interagi avec $\mathrm{HClO}$ produit électrochimiquement est illustré par les images obtenues par microscopie électronique à balayage (Fig. 4). Les images correspondent à un film $\mathrm{SnO}_{2}$ polarisé dans de l'eau de mer naturelle pendant six heures en présence de BSA. La séparation entre surface traitée et non traitée est nette. Il y a formation d'un film mince mais bien couvrant, à la surface duquel on voit la présence d'agrégats répartis de manière plus ou moins régulière. Le spectre EDX révèle la présence des pics des éléments chlore, brome et magnésium (Fig. 5). A noter l'importance du signal dû au brome par rapport à celui du chlore, alors que les concentrations respectives de ces éléments dans l'eau de mer sont de $7,7 \times 10^{-4}$ et 0,47 mol. $\mathrm{kg}^{-1}$. Concernant le calcium, il n'est pas possible de conclure quant à sa présence ou non, car le pic correspondant est masqué par le signal de l'étain $(3,7 \mathrm{keV})$. On trouve également les pics du carbone et du soufre, qui sont la signature de la matière organique agglomérée à la surface. La détection 
de l'élément brome dans le film déposé provient de l'oxydation des ions bromure naturellement présents dans l'eau de mer. Visà-vis de l'interaction avec les protéines, les ions bromure et chlorure jouent un rôle symétrique via la formation des espèces $\mathrm{HOCl}$ et $\mathrm{HOBr}$, qui selon la littérature, peuvent réagir avec les protéines pour former des liaisons de type $>\mathrm{N}-\mathrm{Cl}[19,20]$ et $>\mathrm{N}-\mathrm{Br}[21,22]$. L'oxydation des ions bromure, via la formation de $\mathrm{HBrO}$, peut conduire à la formation de groupements bromamine dans la matière organique à la surface, comme le font les ions chlorure avec la formation de fonctions chloramine. La présence simultanée d'ions bromure et chlorure dans l'eau de mer permet d'envisager la formation simultanée de fonctions chloramine et bromoamine [23], situation favorable au renforcement d'un possible effet antibactérien.

La réactivité de $\mathrm{HOCl}$ et $\mathrm{HOBr}$ vis-à-vis des chaînes latérales des acides aminés formant les protéines a fait l'objet de nombreux travaux décrits dans une revue récente [24]. Les acides hypohalogéneux réagissent principalement avec des acides aminés soufrés, tels que la cystéine et la méthionine [25], et avec ceux portant des fonctions amine, tels que la lysine [26]. Les études cinétiques montrent, pour la plupart des systèmes étudiés, des vitesses de réaction beaucoup plus élevées pour $\mathrm{HOBr}$ que pour $\mathrm{HOCl}$ [24]. La plus grande réactivité de $\mathrm{HOBr}$ par rapport à $\mathrm{HOCl}$ fait du premier un candidat de choix pour modifier chimiquement des protéines. Du fait de sa composition, l'oxydation électrochimique de l'eau de mer conduit naturellement à la co-production de $\mathrm{HOCl}$ et $\mathrm{HOBr}$, combinant ainsi l'action oxydante des deux acides.

Pour montrer le caractère antibactérien d'une surface $\mathrm{SnO}_{2}$ ainsi modifiée [27], nous présentons ici les observations effectuées par microscopie à fluorescence sur des échantillons de films $\mathrm{SnO}_{2}$ polarisés anodiquement dans de l'eau de mer naturelle additionnée ou non de protéines. À titre de comparaison, la figure 6a montre la colonisation d'une surface de $\mathrm{SnO}_{2}$ par E. coli, surface initialement polarisée à $+1,5 \mathrm{~V} / \mathrm{ECS}$ en l'absence de BSA. L'image obtenue est très semblable à celle d'un film $\mathrm{SnO}_{2}$ non traitée (Fig. 1c), montrant une forte colonisation. La polarisation anodique en l'absence de BSA n'est pas suffisante pour produire un effet antibactérien. C'est ce que démontre la figure $6 \mathrm{~b}$, pour laquelle, après polarisation à $+1,5$ V/ECS dans de l'eau de mer en pré-
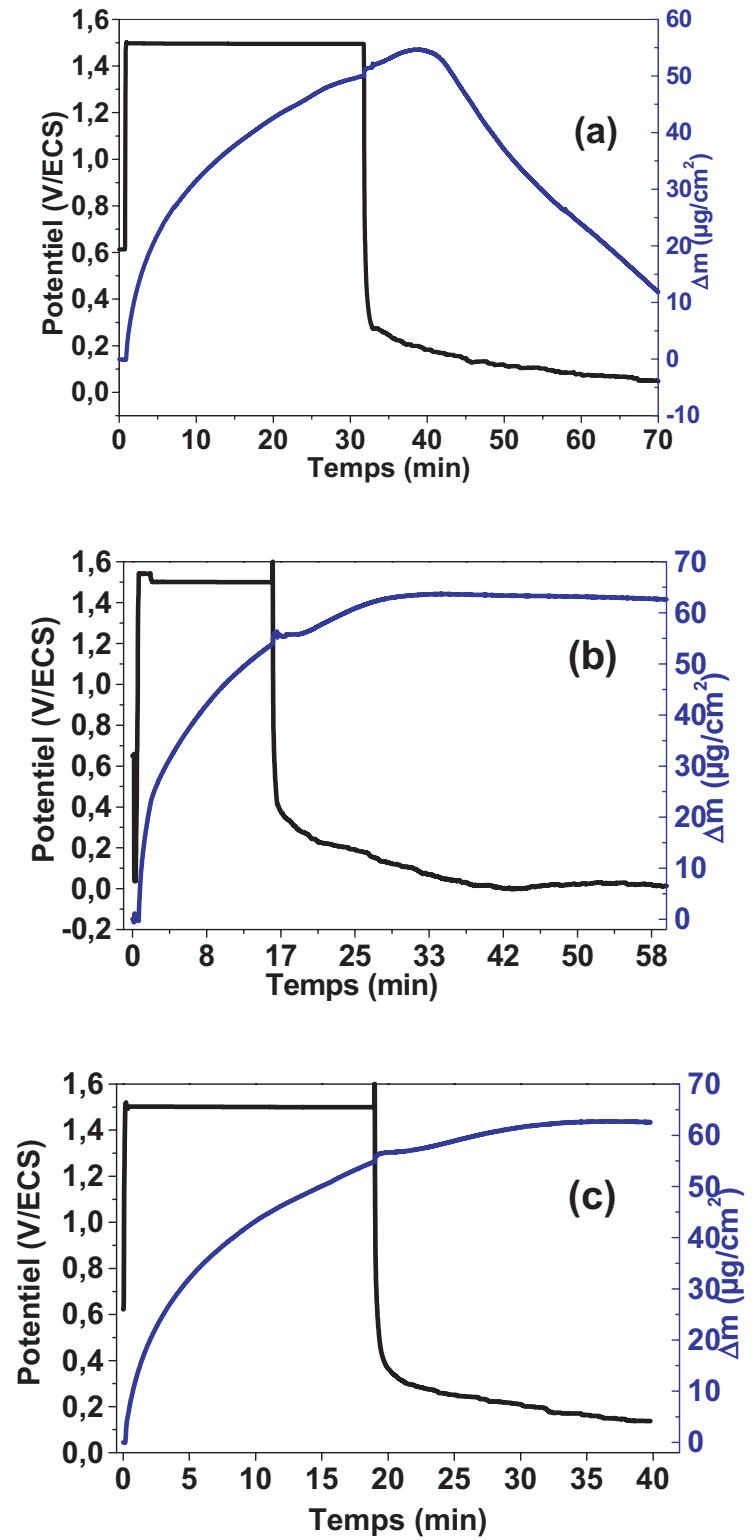

Fig. 3. Réponses chronoélectrogravimétriques d'un film $\mathrm{SnO}_{2}$ en circuit ouvert, puis maintenu à $+1,5 \mathrm{~V} / \mathrm{ECS}$, puis à nouveau en circuit ouvert, en présence de BSA à $0,3 \%$ dans : a) $0,5 \mathrm{M} \mathrm{NaCl}$, b) $\mathrm{NaCl}+\mathrm{CaCl}_{2}(34,85 \mathrm{~g} / \mathrm{L}+1,15 \mathrm{~g} / \mathrm{L})$, c) $\mathrm{NaCl}+\mathrm{MgCl}_{2}(30,8 \mathrm{~g} / \mathrm{L}+5,2 \mathrm{~g} / \mathrm{L})$.

Fig. 3. Mass variation vs. time of a $\mathrm{SnO}_{2}$ film in open circuit, then held at $+1.5 \mathrm{~V} / \mathrm{SCE}$, then again in open circuit, in the presence of $0.3 \% \mathrm{BSA}$ in: a) $0.5 \mathrm{M} \mathrm{NaCl}$, b) $\mathrm{NaCl}+\mathrm{CaCl}_{2}$ $(34.85 \mathrm{~g} / \mathrm{L}+1.15 \mathrm{~g} / \mathrm{L}), \mathrm{c}) \mathrm{NaCl}+\mathrm{MgCl}_{2}(30.8 \mathrm{~g} / \mathrm{L}+5.2 \mathrm{~g} / \mathrm{L})$.

sence de $0,1 \%$ de BSA $(1 \mathrm{mg} / \mathrm{mL})$, la surface est exempte presque totalement de bactéries. Ainsi, dans l'eau de mer, on bénéficie de l'effet conjoint des groupements chloramine et bromamine vis-à-vis des bactéries.

\section{Conclusion}

Les résultats présentés ici démontrent que l'anodisation d'un film de $\mathrm{SnO}_{2}$ dans un milieu chloruré, comme l'eau de mer naturelle, 


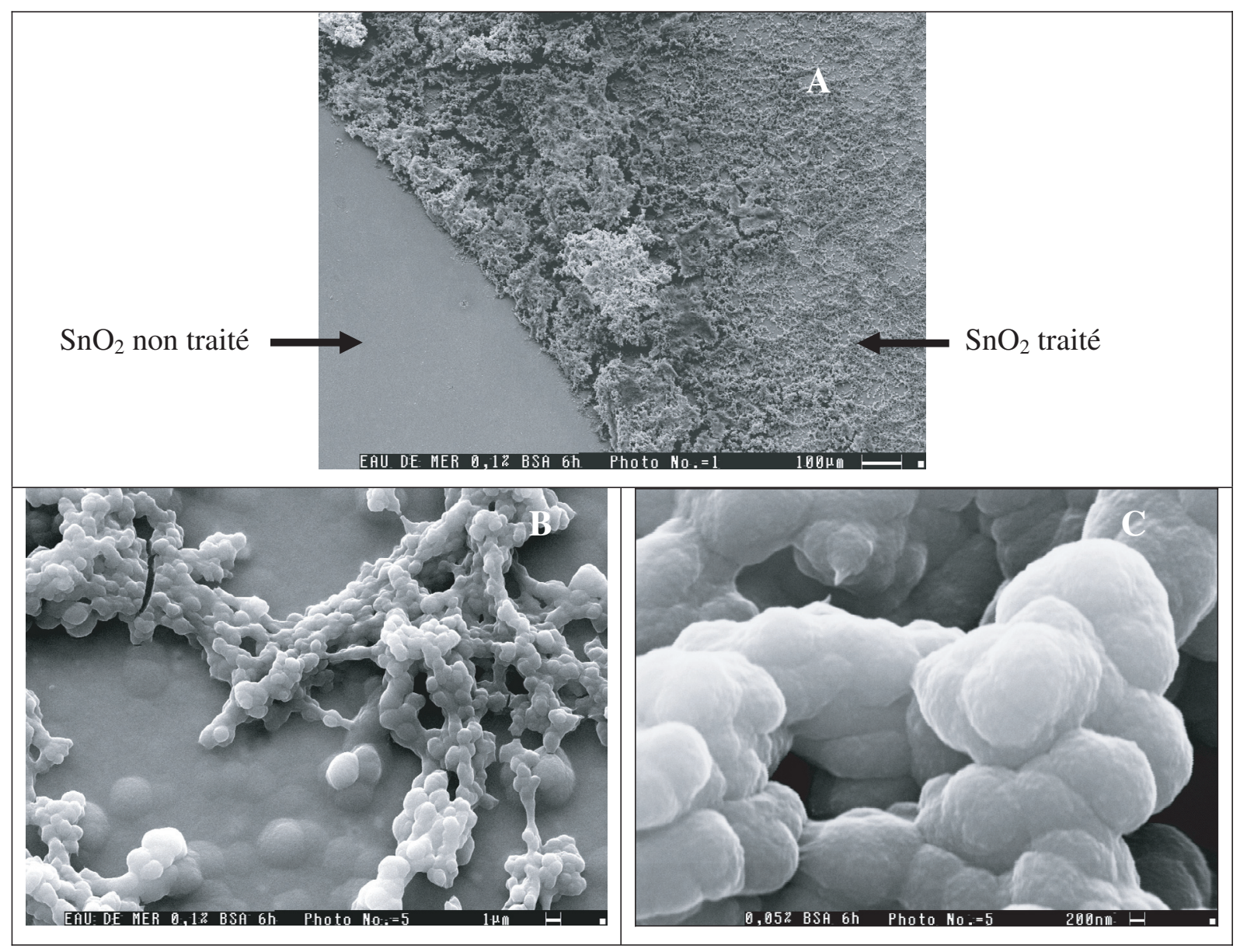

Fig. 4. Observations par microscopie électronique à balayage de la surface de $\mathrm{SnO}_{2}$ polarisée dans l'eau de mer naturelle pendant 6 heures en présence de BSA à la concentration de $0,1 \%$ a) et b), de $0,05 \% \mathrm{c}$ ).

Fig. 4. Scanning electron microscopy images of a $\mathrm{SnO}_{2}$ surface polarised for 6 hours in natural seawater in the presence of $0.1 \%$ BSA a) and b), $0.05 \%$ BSA c).

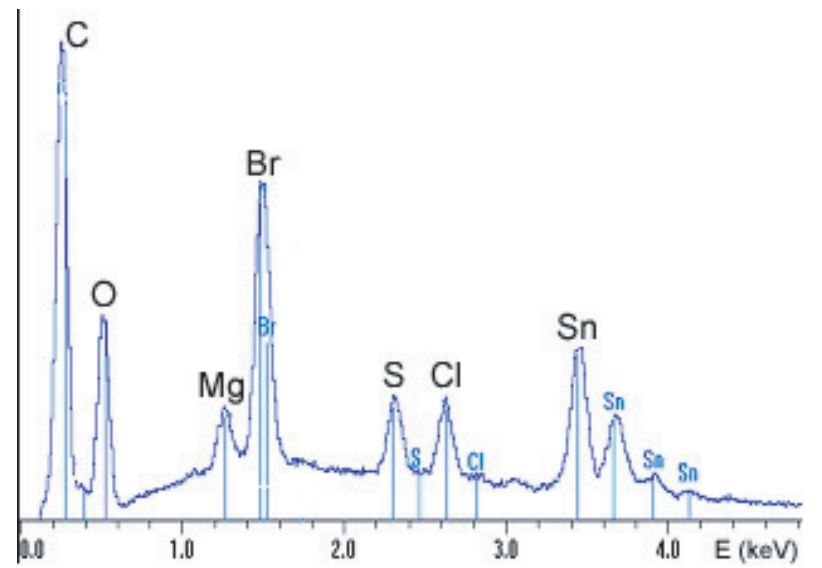

Fig. 5. Spectre EDX d'un film $\mathrm{SnO}_{2}$ polarisé à $+1,5 \mathrm{~V} / \mathrm{ECS}$ pendant 6 heures dans de l'eau de mer naturelle en présence de BSA à $0,1 \%$.

Fig. 5. EDX spectrum of a $\mathrm{SnO}_{2}$ film polarised at $+1.5 \mathrm{~V} / \mathrm{SCE}$ for 6 hours in natural seawater in the presence of $0.1 \%$ BSA.

en présence de l'albumine BSA constitue un traitement efficace pour obtenir une pro- tection antibactérienne. Le film organique formé sur la surface n'acquiert un caractère antibactérien qu'après une étape de polarisation anodique en présence de protéines. L'agrégation et le dépôt en quantité des molécules de BSA ayant fixé du chlore et/ou du brome résultent de la production des acides hypochloreux et hypobromeux par oxydation électrochimique des ions chlorure et bromure, présents dans l'eau de mer. La modification chimique de la BSA, au niveau des chaînes latérales de certains acides aminés, est d'autant plus efficace que la quantité de BSA au voisinage de l'électrode est petite devant les quantités de $\mathrm{HClO}$ et $\mathrm{HBrO}$ produites.

Les résultats obtenus prouvent clairement que la croissance d'Escherichia coli sur la surface de $\mathrm{SnO}_{2}$ électrochimiquement modifiée en présence de la protéine BSA et $\mathrm{d}$ 'ions chlorure et/ou bromure est très inférieure à celle obtenue sur les échantillons traités électrochimiquement en absence de 


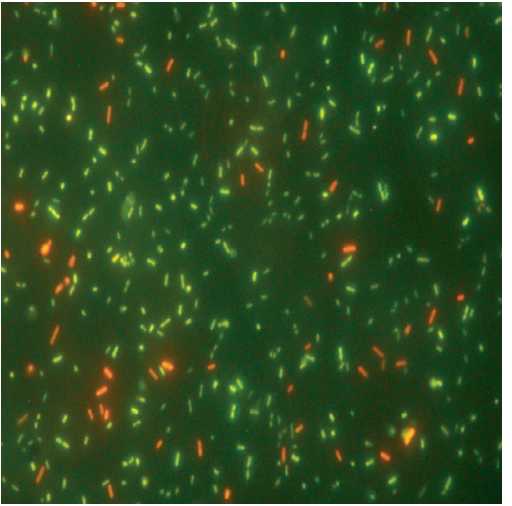

(a)

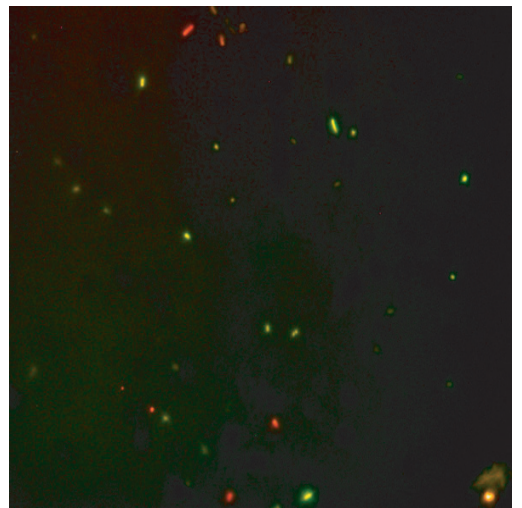

(b)

Fig. 6. a) Image de $E$. coli obtenue par microscopie à fluorescence pour un film $\mathrm{SnO}_{2}$ polarisé dans l'eau de mer à $+1,5 \mathrm{~V} / \mathrm{ECS}$, en l'absence de BSA, pendant $1 \mathrm{~h} 30 ; \mathrm{b}$ ) Image obtenue par microscopie à fluorescence après 2 heures de polarisation à $+1,5 \mathrm{~V} / \mathrm{ECS}$ dans l'eau de mer en présence de BSA à $0,1 \%$.

Fig. 6. a) Fluorescence microscopy images of E. coli of a $\mathrm{SnO}_{2}$ film, polarised at $+1.5 \mathrm{~V} / \mathrm{SCE}$ in seawater for 1 h30 in the absence of BSA; $b$ ) After being polarised at +1.5 V/SCE for 2 hours in seawater in the presence of $0.1 \%$ BSA.

l'albumine et sur ceux simplement immergés dans une solution contenant de l'albumine et des ions chlorure et bromure. Ces résultats précisent les propriétés antibactériennes acquises par ces surfaces de $\mathrm{SnO}_{2}$ traitées électrochimiquement en eau de mer naturelle; ces propriétés peuvent être attribuées à la présence conjointe de fonctions chloramine et bromamine immobilisées au sein du dépôt organique.

\section{Remerciements}

Ce travail a été réalisé dans le cadre du programme PNIR «Biofilms » du CNRS.

\section{Références}

[1] F. Moran, Matér. Tech. 9-10 (2002) 9

[2] E.L. Thomas, M.B. Grisham, M.M. Jefferson, Meth. Enzymol. 132 (1986) 569

[3] M.N.B. Momba, T.E. Cloete, S.N. Venter, R. Kfir, Water Res. 33 (1999) 2937

[4] M.N.B. Momba, T.E. Cloete, S.N. Venter, R. Kfir, Water Sci. Technol. 38 (1998) 283

[5] A.V. Peskin, C.C. Winterbourn, Free Rad. Biol. Med. 40 (2006) 45

[6] A.V. Peskin, C.C. Winterbourn, Free Rad. Biol. Med. 35 (2003) 1252

[7] A.V.Peskin, C.C. Winterbourn, Free Rad. Biol. Med. 30 (2001) 572

[8] A.L.P. Chapman, R. Senthilmohan, C.C. Winterbourn, A.J. Kettle, Arch. Biochem. Biophys. 377 (2000) 95

[9] C.C. Winterbourn, S.O. Brennan, Biochem. J. 326 (1997) 87
[10] C.L. Hawkins, M.J. Davies, Biochem. J. 332 (1998) 617

[11] C.L. Hawkins, M.J. Davies, Biochem. J. 340 (1999) 539

[12] C.L. Hawkins, M.D. Rees, M.J. Davies, FEBS Lett. 510 (2002) 41

[13] S. Haskouri, H. Cachet, C. Debiemme-Chouvy, R. Warocquier-Clerout, M.D. Nagel, Matér. Tech. 93 (7-9) (2005) 135

[14] C. Debiemme-Chouvy, S. Haskouri, G. Folcher, H. Cachet, Langmuir 23 (2007) 3873

[15] K. Bizet, C. Gabrielli, H. Perrot, Appl. Biochem. Biotech. 89 (2000) 139

[16] H. Cachet, S. Haskouri, G. Folcher, B. Tribollet, D. Festy, Matér. Tech. 7-8 (2004) 9

[17] S. Haskouri, H. Cachet, J.L. Duval, C. Debiemme-Chouvy, Electrochem. Comm. 8 (2006) 1115

[18] C. Poleunis, C. Rubio, C. Compère, P. Bertrand, Appl. Surf. Sci. 203-204 (2003) 693

[19] A.V.Peskin, C.C. Winterbourn, Free Rad. Biol. Med. 40 (2006) 45

[20] C.L. Hawkins, M.J. Davies. Chem. Res. Toxicol. 18 (2005) 1600

[21] A.C. Carr, J.J.M. van den Berg, C.C. Winterbourn, Biochim. Biophys. Acta 1392 (1998) 254

[22] W. Wu, Y. Chen, A. d'Avignon, S.L. Hazen, Biochemistry 38 (1999) 3538

[23] C. Debiemme-Chouvy, S. Haskouri, H. Cachet, Appl. Surf. Sci. 253 (2007) 5506

[24] D.I. Pattison, M.J. Davies, Curr. Medic. Chem. 13 (2006) 3271

[25] C.L. Hawkins, M.J. Davies, Free Rad. Biol. Med. 39 (2005) 900

[26] C.L. Hawkins, M.J. Davies, Biochem. J. 332 (1998) 617

[27] S. Haskouri, H. Cachet, J.L. Duval, C. Debiemme-Chouvy, Electrochem. Comm. 8 (2006) 1115 